\title{
Microsurgical treatment of unruptured middle cerebral artery aneurysms: a large, contemporary experience
}

\author{
Eric S. Nussbaum, MD, ${ }^{1}$ Michael T. Madison, MD, ${ }^{1}$ James K. Goddard, MD, ${ }^{1}$ Jeffrey P. Lassig, MD, ${ }^{1}$ \\ Kevin M. Kallmes, MA, ${ }^{2}$ and Leslie A. Nussbaum, MD, PhD'
}

${ }^{1}$ National Brain Aneurysm Center, Twin Cities, Minnesota; and 2Duke University Law School, Durham, North Carolina

OBJECTIVE Advances in endovascular therapy for the treatment of middle cerebral artery (MCA) aneurysms have led to scrutiny of its benefits compared with microsurgical repair. To provide information regarding complication rates and outcomes, the authors reviewed the results of a large series of unruptured MCA aneurysms treated with open microsurgery.

METHODS The authors included all patients who underwent surgical repair of an unruptured MCA aneurysm between 1997 and 2015. All surgical procedures, including clipping, wrapping, bypass, and parent artery occlusion, were performed by a single neurosurgeon. Aneurysm occlusion was assessed using intraoperative digital subtraction angiography (DSA) or DSA and indocyanine green videoangiography in all cases. Postoperatively, all patients were monitored in a neurointensive care unit overnight. Clinical follow-up was scheduled for 2-4 weeks after surgery, and angiographic follow-up was performed in those patients with subtotally occluded aneurysms at 1, 2, and 5 years postoperation.

RESULTS The authors treated 750 unruptured MCA aneurysms in 716 patients: $649(86.5 \%)$ aneurysms were small, 75 $(10.0 \%)$ were large, and $26(3.5 \%)$ were giant. Most aneurysms $(n=677,90 \%)$ were treated by primary clip reconstruction. The surgical morbidity rate was $2.8 \%$, and the mortality rate was $0 \%$. Complete angiographic aneurysm occlusion was achieved in $92.0 \%$ of aneurysms. At final follow-up, 713 patients had a modified Rankin Scale (mRS) score of 0, 2 patients had an mRS score of 2 or 3 , and 1 had an mRS score of 4 .

CONCLUSIONS In high-volume centers, microsurgical management of MCA aneurysms can be performed with very low morbidity rates. Currently, microsurgical repair appears to be a highly effective method of treating MCA aneurysms.

https://thejns.org/doi/abs/10.3171/2018.1.JNS172466

KEYWORDS aneurysm; clip; coil; endovascular; middle cerebral artery; unruptured; vascular disorders

$\mathrm{T}$ HE introduction of the Guglielmi Detachable Coil system in the early 1990s ushered in the era of endovascular treatment for intracranial aneurysms. ${ }^{10}$ Thereafter, the role of endovascular therapy in the management of these lesions expanded with the introduction of balloon remodeling, stent-assisted coiling, and, most recently, flow-diverting stents. Middle cerebral artery (MCA) bifurcation aneurysms are situated peripherally and typically have a wide neck that incorporates the origin of one or both $\mathrm{M}_{2}$ segments. As a result of this configuration, MCA aneurysms largely remained in the domain of surgical repair, 1,18,19,21,24,26,28 Increasingly, however, endovascular therapy has been used as an option for treating aneurysms in this location. ${ }^{2-4,9,11,13,15,16,23,30,33}$ The optimal treatment modality, however, is controversial. ${ }^{2,25,28}$ To provide information regarding surgical complication rates and treatment results for MCA bifurcation aneurysms, we reviewed the outcomes of 750 unruptured MCA aneurysms treated with open microsurgery by a single surgeon. To our knowledge, this represents the largest such series reported to date.

\section{Methods}

\section{Patient Selection and Aneurysm Characteristics}

All patients who were referred to the National Brain Aneurysm Center for treatment of an unruptured MCA aneurysm and underwent open surgical treatment of their lesion by a single surgeon between 1997 and 2015 were included in this retrospective case series based on a review of prospectively collected data. Preoperative catheter angiography was performed in all cases. Aneurysm size

ABBREVIATIONS DSA = digital subtraction angiography; DVT = deep venous thrombosis; $M C A=$ middle cerebral artery; $m R S=$ modified Rankin Scale; SAH = subarachnoid hemorrhage.

SUBMITTED October 2, 2017. ACCEPTED January 10, 2018.

INCLUDE WHEN CITING Published online June 22, 2018; DOI: 10.3171/2018.1.JNS172466. 
was measured based on maximum dome width using the following scale: small, $<10 \mathrm{~mm}$; large, $10-25 \mathrm{~mm}$; giant, $>25 \mathrm{~mm}$. At our clinic, the principal decision to proceed with surgery is based on the estimated lifetime risk of aneurysm rupture compared to the anticipated risk of aneurysm repair, taking into consideration patient age, medical comorbidities, family or personal history of subarachnoid hemorrhage (SAH), psychological impact of aneurysm presence, and input from endovascular colleagues regarding the ease of endovascular treatment. All cases were included in a prospective database that included preoperative notes, operative reports, and postoperative outpatient notes. Imaging studies were reviewed for patients with a surgical complication. Lastly, to further investigate a comparison between surgical and endovascular treatment of "routine" MCA aneurysms, we performed a subgroup analysis of the small, saccular, noncoiled aneurysms.

\section{Surgical Technique}

We approached the aneurysms utilizing a standard pterional craniotomy, unless otherwise indicated. The intradural portion of all surgeries, including opening of the sylvian fissure, dissection, and clipping of the aneurysm, was performed under microsurgical magnification. No neuromonitoring was used, and all operations after 2001 (the most recent 701 surgeries) were performed without the inclusion of residents or fellows. Subtotal occlusion was undertaken when attempted complete occlusion resulted in narrowing of the parent artery or perforator compromise, or when calcification or atheroma precluded safe clip placement at the true aneurysm neck. When subtotal occlusion was performed, the area of the aneurysm that was left untreated was subsequently wrapped in all cases with muslin-type gauze, cotton, or Gore-Tex. Temporary clipping was only utilized for large or giant aneurysms to diminish aneurysm turgor and decrease the risk of aneurysm rupture during manipulation. Temporary clipping was used also in selected cases of previously coiled aneurysms during clipping of the aneurysm neck below the coils or during opening of the aneurysm to extract coils.

\section{Aneurysm Occlusion on Digital Subtraction Angiography}

Every patient underwent intraoperative digital subtraction angiography (DSA) by a nonblinded neuroradiologist to confirm appropriate clipping and to exclude the presence of local vascular compromise. Beginning in July 2010, the use of indocyanine green videoangiography (ICG) was added to provide complementary data in the remaining cases $(n=370)$. A comparison of ICG and DSA data was previously published for a portion of these cases and is not recorded here. ${ }^{7}$ For patients treated by clipping or bypass/sacrifice, the radiologist rated aneurysm occlusion on a percentage basis; in retrospective analysis, $100 \%$ was considered "complete occlusion/obliteration," 90\%99\% was considered "residual neck," and 0\%-89\% was considered "incomplete occlusion."

\section{Perioperative Management and Follow-Up Characteristics}

All surgeries were performed with the patient under general endotracheal anesthesia using barbiturate anesthe- sia and mild hypothermia $\left(34^{\circ} \mathrm{C}\right)$. Patients received mannitol (1 g/kg), Decadron (4-10 mg intravenously), and a loading dose of either fosphenytoin or levetiracetam. Intravenous antibiotics were administered preoperatively and continued for 24 hours postoperatively. All patients were monitored overnight in a neurointensive care unit, and systolic blood pressure was kept below $140 \mathrm{~mm} \mathrm{Hg}$. Patients were mobilized early, including the night of surgery. Patients were typically discharged between 48 and 96 hours after surgery, unless complications occurred in this period or circumstances precluded discharge. Postoperative infection was only recorded if intravenous antibiotics or surgical reexploration were required, and the database does not account for cases in which the patient's primary care physician may have prescribed oral antibiotics for a superficial wound infection. Patients only underwent delayed angiographic examination if they had subtotal occlusion of their aneurysm on intraoperative DSA. Patients' modified Rankin Scale (mRS) scores were recorded by an unblinded author or nurse practitioner at follow-up office visits. If complications occurred and patients underwent multiple follow-up appointments, mRS scores were determined on the ultimate clinic visit. Clinical follow-up was planned between 2 and 4 weeks after surgery, but cases with subtotal occlusion underwent angiographic follow-up at 1,2 , and 5 years after surgery as well.

\section{Results}

\section{Patient and Aneurysm Characteristics}

Altogether, there were $750 \mathrm{MCA}$ aneurysms in 716 patients (512 females and 204 males; mean age 43 years, range 6-79 years). Of these patients, a single MCA aneurysm was treated in 687 patients (95.9\%), bilateral MCA aneurysms in 25 patients (3.5\%), 3 MCA aneurysms in 3 patients $(0.4 \%)$, and $4 \mathrm{MCA}$ aneurysms in 1 patient $(0.1 \%)$. Of the 750 unruptured MCA aneurysms treated with open microsurgical techniques, 649 (86.5\%) were small, 75 (10.0\%) were large, and 26 (3.5\%) were giant. Twenty-four aneurysms (3.2\%) had been previously coiled, of which 19 were small, 3 were large, and 2 were giant lesions. Thirtyfour patients (4.7\%) had a history of SAH, and 92 (12.8\%) had aneurysms in locations other than the MCA and its branches (Table 1). Of the 750 MCA aneurysms, 169 were reported previously as part of an analysis of unruptured intracranial aneurysms..$^{21}$

\section{Surgical Treatment}

All aneurysms were approached through a standard pterional craniotomy, apart from 10 giant aneurysms that required an orbitozygomatic approach and 18 peripherally located aneurysms that required extension of the pterional craniotomy. The mean surgical time from incision to completion of closure was 90 minutes (range 59-156 minutes for simple clipping cases, 192-450 minutes for cases requiring revascularization). Most aneurysms were treated with direct surgical clipping (677/750, 90.3\%). Parent artery sacrifice and surgical revascularization were used in 52 cases $(7.0 \%)$, particularly when managing giant or fusiform lesions. Twenty-one aneurysms (3.0\%) were treated with wrapping alone. Of the 24 previously coiled 
TABLE 1. Patient and aneurysm characteristics

\begin{tabular}{|c|c|}
\hline Variable & Value \\
\hline \multicolumn{2}{|l|}{ Background characteristics } \\
\hline No. of patients & 716 \\
\hline \multicolumn{2}{|l|}{ Sex } \\
\hline Male & $204(28.5 \%)$ \\
\hline Female & $512(71.5 \%)$ \\
\hline Mean age, yrs (range) & $43(6-79)$ \\
\hline \multicolumn{2}{|l|}{ No. of MCA aneurysms treated } \\
\hline 1 & $687(95.9 \%)$ \\
\hline 2 (bilateral) & $25(3.5 \%)$ \\
\hline 3 & $3(0.4 \%)$ \\
\hline 4 & $1(0.1 \%)$ \\
\hline $\begin{array}{l}\text { No. of patients w/ additional aneurysms not } \\
\text { involving the MCA or its branches* }\end{array}$ & $92(12.8 \%)$ \\
\hline Anterior communicating artery & $45(6.3 \%)$ \\
\hline PCoA or anterior choroidal artery & $18(2.5 \%)$ \\
\hline Ophthalmic artery & $8(1.1 \%)$ \\
\hline Basilar artery & $3(0.4 \%)$ \\
\hline Other† & $18(2.5 \%)$ \\
\hline History of SAH & $34(4.7 \%)$ \\
\hline From aneurysm that was coiled & $28(3.9 \%)$ \\
\hline From surgically treated aneurysm & $6(0.8 \%)$ \\
\hline \multicolumn{2}{|l|}{ Presenting aneurysm characteristics } \\
\hline No. of aneurysms & 750 \\
\hline \multicolumn{2}{|l|}{ Previously coiled } \\
\hline Yes & $24(3.2 \%)$ \\
\hline No & $726(96.8 \%)$ \\
\hline \multicolumn{2}{|l|}{ Size } \\
\hline Small & $649(86.5 \%)$ \\
\hline Large & $75(10.0 \%)$ \\
\hline Giant & $26(3.5 \%)$ \\
\hline \multicolumn{2}{|l|}{ Size of coiled aneurysms } \\
\hline Small & $19(2.5 \%)$ \\
\hline Large & $3(0.4 \%)$ \\
\hline Giant & $2(0.3 \%)$ \\
\hline
\end{tabular}

PCoA = posterior communicating artery.

* Except as otherwise noted, aneurysms in these locations were clipped during the same procedure as the MCA aneurysms reported in this series.

† These aneurysms were monitored, coiled, or treated surgically after a separate craniotomy.

aneurysms, $9(38 \%)$ were treated by primary clipping below the coil mass, $11(46 \%)$ by distal bypass with vascular sacrifice, 2 (8\%) by coil extraction followed by clipping, and $2(8 \%)$ by simple wrapping.

\section{Occlusion Rates and Complications}

In aneurysms treated with clipping or bypass/sacrifice $(n=729)$, complete occlusion based on intraoperative angiographic examination was achieved in 671 aneurysms (92.0\%), and residual neck was left in 58 aneurysms (8.0\%). No aneurysms had incomplete occlusion (0.0\%; Table 2$)$. Intraoperative rupture occurred in 3 patients $(0.4 \%)$. In
TABLE 2. Treatments, outcomes, and complications for 716 patients with 750 aneurysms

\begin{tabular}{|c|c|}
\hline Variable & Value \\
\hline No. of aneurysms & 750 \\
\hline \multicolumn{2}{|l|}{ Treatment } \\
\hline Clipping & $677(90.3 \%)$ \\
\hline Bypass & $52(7.0 \%)$ \\
\hline Wrapping & $21(3.0 \%)$ \\
\hline \multicolumn{2}{|l|}{ Occlusion } \\
\hline $100 \%$ & $671(89.5 \%)$ \\
\hline $95-99 \%$ & $51(7.0 \%)$ \\
\hline $90-94 \%$ & $7(1.0 \%)$ \\
\hline NA (wrapped) & $21(3.0 \%)$ \\
\hline No. of patients & 716 \\
\hline \multicolumn{2}{|l|}{ mRS score } \\
\hline 0 & $713(99.6 \%)$ \\
\hline 1 & $0(0.0 \%)$ \\
\hline 2 & $1(0.1 \%)^{*}$ \\
\hline 3 & $1(0.1 \%) \dagger$ \\
\hline 4 & $1(0.1 \%) \ddagger$ \\
\hline Intraop rupture & $3(0.4 \%)$ \\
\hline \multicolumn{2}{|l|}{ Complications } \\
\hline SVG thrombosis & $1(0.1 \%)$ \\
\hline Perforating artery injury & $2(0.3 \%)$ \\
\hline Periop seizure & $4(0.6 \%)$ \\
\hline Transient ND & $4(0.6 \%)$ \\
\hline DVT§ & $6(0.8 \%)$ \\
\hline Wound infection & $2(0.3 \%)$ \\
\hline Pterional fullness/discomfort & $11(1.5 \%)$ \\
\hline
\end{tabular}

DVT = deep venous thrombosis; $\mathrm{NA}=$ not applicable; $\mathrm{ND}=$ neurological deficit; SVG = saphenous vein graft.

* Case 15 in Table 3. This patient had an initial mRS score of 3 at the 30-day follow-up, which subsequently decreased to an mRS score of 2 at the 6 -month and 5 -year follow-up.

† Case 16 in Table 3. This patient had an initial mRS score of 4 at the 30 - and 90-day follow-ups, which subsequently decreased to an mRS score of 3 at the 6-month and 3-year follow-ups.

$\ddagger$ Case 4 in Table 3. This patient had an initial mRS score of 5 at the 30 - and 90-day follow-ups, which subsequently decreased to an mRS score of 4 at the 1 - and 4-year follow-ups.

$\S$ Case 4 in Table 3 . This patient experienced both deep vein thrombosis and a saphenous vein thrombosis, which are reported as separate complications in this table.

one, the rupture was controlled by placing cotton at the rupture site, temporarily clipping the $\mathrm{M}_{1}$ segment, and then finishing the dissection; and in another the aneurysm dome was clipped preliminarily below the rupture site, allowing us to complete the exposure and clipping of the aneurysm neck. The remaining patient was given adenosine while the dissection was completed and the aneurysm clipped. Each patient recovered fully.

\section{Complications}

Treatment-related complications occurred in 20 patients (2.8\%, Tables 2 and 3); 3 of these patients (15\%) 
TABLE 3. Summary of treatment-related complications

\begin{tabular}{|c|c|c|c|c|c|c|c|}
\hline $\begin{array}{l}\text { Case } \\
\text { No. }\end{array}$ & $\begin{array}{l}\text { Age } \\
\text { (yrs), } \\
\text { Sex }\end{array}$ & $\begin{array}{l}\text { Aneurysm } \\
\text { Size }(\mathrm{mm})\end{array}$ & $\begin{array}{l}\text { Presenting } \\
\text { Symptoms }\end{array}$ & Complication & Treatment & Duration & Outcome \\
\hline 1 & $45, \mathrm{~F}$ & 9 & LE pain, swelling & DVT & IVC filter & 6 wks; filter removed & Full recovery \\
\hline 2 & $59, \mathrm{~F}$ & 6 & LE pain, swelling & DVT & IVC filter & 6 wks; filter removed & Full recovery \\
\hline 3 & $41, \mathrm{M}$ & 22 & LE pain, swelling & DVT & Anticoagulation & $6 \mathrm{mos}$ & Full recovery \\
\hline \multirow{2}{*}{4} & $62, \mathrm{M}$ & 42 & LE pain, swelling & DVT & Anticoagulation & $6 \mathrm{mos}$ & $\begin{array}{l}\text { LE pain \& swelling } \\
\text { resolved }\end{array}$ \\
\hline & & 42 & $\begin{array}{l}\text { Hemiplegia, hemi- } \\
\text { sensory loss, vi- } \\
\text { sual field defect, } \\
\text { mild neglect }\end{array}$ & $\begin{array}{l}\text { Delayed SVG throm- } \\
\text { bosis }\end{array}$ & None & Permanent & $\begin{array}{l}\text { Good cognitive function; } \\
\text { severe permanent } \\
\text { disability requiring } \\
\text { assistance w/ ADLs }\end{array}$ \\
\hline 5 & $55, \mathrm{~F}$ & 15 & LE pain, swelling & DVT & IVC filter \& anticoagulation & $9 \mathrm{mos}$ & Full recovery \\
\hline 6 & $67, \mathrm{M}$ & 12 & LE pain, swelling & DVT & IVC filter \& anticoagulation & $6 \mathrm{mos}$ & Full recovery \\
\hline 7 & $39, \mathrm{~F}$ & 10 & Seizure & Postop seizures & Overnight intubation & $\begin{array}{l}\text { 6-mo anticonvulsant } \\
\text { therapy }\end{array}$ & Resolved \\
\hline 8 & $54, \mathrm{~F}$ & 6 & Seizure & Postop seizures & Lorazepam & $\begin{array}{l}\text { 3-mo anticonvulsant } \\
\text { therapy }\end{array}$ & Resolved \\
\hline 9 & $49, \mathrm{M}$ & 7 & Seizure & Postop seizures & Lorazepam & $\begin{array}{l}\text { 3-mo anticonvulsant } \\
\text { therapy }\end{array}$ & Resolved \\
\hline 10 & $55, \mathrm{~F}$ & 11 & Seizure & Postop seizures & Lorazepam & $\begin{array}{l}\text { 6-mo anticonvulsant } \\
\text { therapy }\end{array}$ & Resolved \\
\hline 11 & $61, F$ & 6 & Dysphasia & Transient NDs & Decadron & $1 \mathrm{wk}$ & Resolved \\
\hline 12 & $58, \mathrm{M}$ & 7 & Dysphasia & Transient NDs & Decadron & 5 days & Resolved \\
\hline 13 & $49, \mathrm{~F}$ & 9 & Hemiparesis & Transient NDs & Decadron & 1 wk & Resolved \\
\hline 14 & $50, \mathrm{~F}$ & 8 & Hemiparesis & Transient NDs & Decadron & 3 days & Resolved \\
\hline 15 & $61, \mathrm{~F}$ & 12 & Hemiparesis & Perforating artery injury & None & Permanent & Mild, permanent \\
\hline 16 & $55, \mathrm{~F}$ & 15 & Hemiparesis & Perforating artery injury & None & Permanent & Severe, permanent \\
\hline 17 & $55, \mathrm{M}$ & 6 & Cellulitis & Wound infection & Antibiotic & 2 wks & Resolved \\
\hline 18 & $52, \mathrm{M}$ & 7 & $\begin{array}{l}\text { Wound fluctuance, } \\
\text { redness }\end{array}$ & Wound infection & $\begin{array}{l}\text { Surgical reexploration \& } \\
\text { IV antibiotics }\end{array}$ & 3 wks & Resolved \\
\hline 19 & $49, \mathrm{~F}$ & 8 & Temporalis fullness & Postop discomfort & Revision & & Resolved \\
\hline 20 & $42, \mathrm{~F}$ & 7 & Temporalis fullness & Postop discomfort & Revision & & Resolved \\
\hline
\end{tabular}

$\mathrm{ADL}$ = activity of daily living; IV = intravenous; IVC = inferior vena cava; $L E=$ left eye.

experienced permanent deficits. One patient with a giant aneurysm measuring more than $4 \mathrm{~cm}$ developed delayed saphenous vein graft thrombosis, the result of which was a severe ischemic injury of his nondominant hemisphere. He survived with good cognitive function but required significant assistance with daily activities; his 30-day follow-up mRS score was 5, but it improved to 4 at 1 year. His postoperative course was also complicated by lower-extremity deep vein thrombosis. A perforating artery was injured in 2 patients: one patient was left with mild hemiparesis and the other with severe, permanent hemiparesis. One of the patients had an mRS score of 3 at the 30-day follow-up that improved to 2 at 6 months, and the other had an mRS score of 4 at the 30-day follow-up that improved to 3 at 6 months. Both injuries occurred in the setting of a very short $\mathrm{M}_{1}$ segment.

Four patients had a single perioperative seizure within 1 hour of emerging from anesthesia. One required reintubation and remained intubated overnight. The other 3 patients were managed conservatively with lorazepam and underwent continuous electroencephalography (EEG) monitoring for 24 hours. After discharge, anticonvulsant therapy was continued for 3 months in 2 cases and 6 months in the other 2 cases; no patient required indefinite anticonvulsant therapy. Four patients noticed immediate transient neurological deficit (dysphasia in 2 cases and mild contralateral motor weakness in 2 cases), which was likely related to local retraction or venous injury. These 4 patients improved quickly and had recovered by their first postoperative visit, 7-10 days after surgery. Six patients developed deep venous thrombosis (DVT) during the 1st postoperative month. Two were managed with placement of an inferior vena cava filter, 2 with intravenous anticoagulation alone, and 2 with both. These 6 cases resolved without further complications.

Two patients $(0.3 \%)$ developed wound infections that required intravenous antibiotics or surgical reexploration; infections that did not require these measures were not reported in our database. One patient with superficial cellulitis was treated with intravenous antibiotic therapy alone. 
The other patient underwent early reexploration with irrigation of the wound, and the bone flap was left in place. The patient received 3 weeks of intravenous antibiotic therapy, and the infection cleared.

\section{Muscle Discomfort and Cosmetic Issues}

While complaints of "fullness" and/or discomfort in the region of the temporalis muscle were common immediately after surgery, this complaint was transient in most patients. Eleven patients (1.5\%) consistently reported fullness and discomfort to a level that required consultation with a plastic surgeon. In 9 of these patients, the discomfort was not bothersome enough to merit intervention. Two patients, however, underwent revision surgery in conjunction with a craniofacial plastic surgeon. In these cases, all hardware was removed, bony irregularities or defects were covered with bone cement, and the temporalis muscle was recontoured and resuspended. Although both patients stated that they were satisfied with the cosmetic outcome, 1 patient reported some fullness that produced an asymmetrical appearance compared with the unoperated side.

\section{Subgroup Analysis of Small, Routine Aneurysms}

We performed a subgroup analysis of the small, saccular, noncoiled aneurysms $(n=649)$. For these 649 aneurysms, complications occurred in $12(1.8 \%)$ cases, although none resulted in lasting deficits; the follow-up mRS score was 0 in all cases (100\%). However, it is important to consider the potential selection bias of these data when reflecting on these results.

\section{Follow-Up and Patient Outcomes}

Clinical follow-up was evaluated in all 716 patients within 30 days postoperation. The mean follow-up time was 8.5 years (range 6 months to 17 years). Cases with subtotal occlusion also underwent angiographic follow-up at 1,2 , and 5 years after surgery. During the follow-up period, no patients suffered bleeding from the treated aneurysm, and no patients died (0\%). The average mRS score at follow-up was 0 (range $0-4$ ); 713 patients had an mRS score of 0,2 patients had a score of 2 or 3 , and 1 patient had a score of 4 (Table 2). At follow-up, one fusiform $\mathbf{M}_{1}$ aneurysm had enlarged considerably and was therefore treated with an endovascular stent/coil approach.

\section{Discussion}

\section{Management of MCA Aneurysms}

As an "early adopter" of endovascular options, our center advocates strongly for the judicious use of minimally invasive therapies when outcomes are demonstrably comparable to or better than those achieved by microsurgery. Nevertheless, the use of endovascular therapy is expanding in many centers based on the assumption that minimizing invasiveness should correspondingly improve outcomes, even in the absence of evidence that such therapy is as safe as more traditional surgical options. It is therefore important to establish the durability of occlusion and the complication rates associated with both open surgical and endovascular treatment options. As a result, we are reporting our experience with the surgical management of MCA aneurysms in an unselected series of patients treated during the "endovascular era."

Multiple authors have described series of MCA aneurysms treated surgically, often as part of larger reported studies. $1,5,6,8,12,17-22,26,27,29,31,32,34$ In general, outcomes have been favorable, with excellent long-term durability associated with surgical repair of these lesions..$^{21,28}$ In the hands of experienced microvascular surgeons managing intracranial aneurysms at high-volume centers, occlusion rates have exceeded 95\%, while major complication rates have been less than $3 \% .{ }^{28}$ The outcomes of the current series are consistent with these findings (Table 4).

\section{Endovascular Treatment}

Most of the large series describing the endovascular treatment of unruptured MCA aneurysms have also reported favorable results. In contrast to the surgical series, however, the rates of major complication have been significant. In a meta-analysis, Brinjikji et al. found that the overall permanent morbidity rate associated with endovascular therapy for unruptured MCA aneurysms was $5.1 \%$, while the rate of complete or near-complete occlusion was $82 \%{ }^{4}$ In a more recent meta-analysis of 26 studies reporting 2207 aneurysms, Smith and colleagues found that the rate of complete occlusion for endovascular therapy slightly exceeded $50 \% .^{28}$

These findings are corroborated by those of individual series. Suzuki et al. treated 115 patients with MCA aneurysms, including 67 unruptured lesions, with detachable coils and achieved a complete occlusion rate of $46 \% .{ }^{30}$ Complications included parent artery thrombosis (3.5\%), thromboembolisms (3.5\%), and aneurysm perforation $(0.9 \%)$. Of the 67 unruptured aneurysms, distal emboli developed in 2 patients, one of whom suffered a delayed and fatal aneurysm rupture. Vendrell and coworkers treated 49 patients with unruptured MCA aneurysms with stent-assisted coiling. Intrastent clots formed in 10 patients (20\%), resulting in 2 cases of permanent neurological deficits. ${ }^{33}$ The overall rate of permanent neurological morbidity was 4.3\%. Furthermore, complete obliteration was achieved in $71 \%$ of their cases, and their rate of delayed recurrence was $14.6 \%$. Quadros et al. treated 59 unruptured MCA aneurysms and achieved a $42 \%$ complete occlusion rate. ${ }^{23}$ Three aneurysms ruptured during treatment (6\%), 10 thromboembolic events (20\%) occurred, and $14.5 \%$ of the aneurysms recanalized over time. Bracard et al. treated 152 MCA aneurysms in 140 patients. ${ }^{3}$ There were 3 perforations (2\%) and 13 thromboembolic events (8.6\%), including a 3.8\% rate of embolic event in unruptured cases and 1 fatal stroke. In a series of 77 unruptured MCA aneurysms, one patient died, the permanent morbidity rate was $3 \%$, and the recurrence rate was $20 \% .{ }^{14}$

\section{Outcomes}

These results were used to suggest that MCA aneurysms can be treated using endovascular therapy safely and with low complication rates. However, the morbidity and mortality rates associated with these endovascular series consistently exceeded those reported by experienced microneurosurgeons. Nonetheless, endovascular treat- 
TABLE 4. Major series of surgically treated MCA aneurysms

\begin{tabular}{|c|c|c|c|c|c|c|}
\hline Authors \& Year & Study Period & No. of Pts & Ruptured, SAH & Length of FU (mos) & $\%$ Good Outcome & Occlusion Rate (\%) \\
\hline Suzuki et al., 2009 & 1961-1981 & $265^{*}$ & $259(97.7 \%)$ & 0 & 85.0 & NR \\
\hline Rinne et al., 1996 & 1977-1992 & 561 & $513(91.4 \%)$ & 12 & 62 (GOS score <2) & NR \\
\hline Ogilvy et al., 1995 & 1985-1989 & 62 & NR & 1 & 82.3 & NR \\
\hline Morgan et al., 2010 & 1989-2009 & 263 & $0(0 \%)$ & 1.5 & 94.7 & NR \\
\hline Nanda et al., 2014 & 1992-2012 & 125 & NR & 19.3 & $83.8(\mathrm{HH}$ grade <2) & 98 \\
\hline $\begin{array}{l}\text { Rodríguez-Hernández } \\
\text { et al., } 2013\end{array}$ & 1997-2010 & 543 & $282(51.9 \%)$ & 46.8 & $\begin{array}{l}\text { SAH: 92.0; UR: } 72.0 \\
\quad \text { (mRS score <2) }\end{array}$ & 98.3 \\
\hline van Dijk et al., 2011 & 2001-2006 & 105 & $77(73.3 \%)$ & 56.4 & 80 (mRS score <2) & 89 \\
\hline Choi et al., 2012 & $2007-2010$ & 125 & $0(0 \%)$ & 22.5 & 100.0 & 95.8 \\
\hline Regli et al., 1999 & 1993-1997 & 30 & $0(0 \%)$ & 0 & NR & 100 \\
\hline Güresir et al., 2011 & 1999-2009 & 271 & $163(60.1 \%)$ & 60 & 71.2 & 98 \\
\hline Taha et al., 2006 & $2001-2004$ & 133 & $53(39.8 \%)$ & 26.7 & SAH: 44; UR: 81 & 81.4 \\
\hline Diaz et al., 2014 & $2005-2010$ & 34 & $9(26.5 \%)$ & 9.02 & 94.1 & 95 \\
\hline Kim et al., 2013 & $2008-2011$ & 78 & $9(11.5 \%)$ & $0.25-6$ & 93.5 & 92 \\
\hline Dammann et al., 2014 & NR & 87 & NR & 12 & NR & 97 \\
\hline Present series & 1997-2015 & 716 & $0(0 \%)$ & 102 & 99.6 & 92.0 \\
\hline
\end{tabular}

FU = follow-up; GOS = Glasgow Outcome Scale; $\mathrm{HH}=$ Hunt and Hess grade; NR = not reported; Pts = patients; UR = unruptured.

Review of large surgical series adapted with permission from Yang W, Huang J: Treatment of middle cerebral artery (MCA) aneurysms: a review of the literature. Chinese Neurosurg J 1:1, 2015. CC BY 4.0 (https://creativecommons.org/licenses/by/4.0/).

* Overall, 413 patients were reviewed in Suzuki et al., but full information on patient outcomes was given only for the 265 patients with single aneurysms.

ment may be preferable for certain populations and situations. When treatment is deemed advisable, endovascular therapy for MCA aneurysms can be given strong consideration in patients older than 70 years, when the aneurysm wall is calcified significantly, or when the geometry of the aneurysm is favorable for coiling or other endovascular therapy, such as narrow-necked aneurysms. However, for many MCA aneurysms, open microsurgery has demonstrably high occlusion rates and low complication rates, and should be considered a primary method of treatment.

\section{Limitations}

The principal limitation of this investigation is the retrospective nature of the data analysis, which introduces greater risk of confounding and bias. The individuals performing intraoperative angiography and determining posttreatment $\mathrm{mRS}$ scores were unblinded. Postoperative de novo aneurysm formation and minor infections were not reported in our database. Furthermore, the operations reported here were performed by a single, experienced microneurosurgeon and generally without the participation of residents, which may not be comparable to other large, academic centers and may limit the generalizability of the data.

\section{Conclusions}

Over the past 2 decades, endovascular therapy has emerged as an important treatment option for intracranial aneurysms, but it should be utilized only when it has complication rates comparable to those of open microsurgery. For unruptured MCA aneurysms, our report suggests that microsurgical clipping results in favorable outcomes more commonly than endovascular treatment, which should be reserved for older patients, those thought to be at high risk of complications from open surgery based on medical comorbidities or aneurysm-related factors, such as heavy wall atheroma, or aneurysms of particular morphologies. However, available data support the use of microsurgical therapy for management of most unruptured MCA aneurysms.

\section{Acknowledgments}

We acknowledge the nursing staff of the National Brain Aneurysm Center for their dedicated assistance with each of these patients, especially Jodi Lowary for her help in collecting and integrating patient data. We also acknowledge Superior Medical Experts for their editing and literature research support.

We received a grant from the United Hospital Foundation in support of this work.

\section{References}

1. Aghakhani N, Vaz G, David P, Parker F, Goffette P, Ozan A, et al: Surgical management of unruptured intracranial aneurysms that are inappropriate for endovascular treatment: experience based on two academic centers. Neurosurgery 62:1227-1235, 2008

2. Blackburn SL, Abdelazim AM, Cutler AB, Brookins KT, Fargen KM, Hoh BL, et al: Endovascular and surgical treatment of unruptured MCA aneurysms: meta-analysis and review of the literature. Stroke Res Treat 2014:348147, 2014

3. Bracard S, Abdel-Kerim A, Thuillier L, Klein O, Anxionnat R, Finitsis S, et al: Endovascular coil occlusion of 152 middle cerebral artery aneurysms: initial and midterm angiographic and clinical results. J Neurosurg 112:703-708, 2010

4. Brinjikji W, Lanzino G, Cloft HJ, Rabinstein A, Kallmes DF: Endovascular treatment of middle cerebral artery aneurysms: a systematic review and single-center series. Neurosurgery 68:397-402, 2011

5. Choi SW, Ahn JS, Park JC, Kwon DH, Kwun BD, Kim CJ: Surgical treatment of unruptured intracranial middle cerebral artery aneurysms: angiographic and clinical outcomes in 143 
aneurysms. J Cerebrovasc Endovasc Neurosurg 14:289294, 2012

6. Dammann P, Schoemberg T, Müller O, Özkan N, Schlamann M, Wanke I, et al: Outcome for unruptured middle cerebral artery aneurysm treatment: surgical and endovascular approach in a single center. Neurosurg Rev 37:643-651, 2014

7. Defillo A, Qureshi M, Nussbaum ES: Indocyanine green videoangiography and intraoperative catheter digital subtraction angiography in the treatment of intracranial aneurysms: a consecutive series of 235 cases. Cureus 6:e203, 2014

8. Diaz OM, Rangel-Castilla L, Barber S, Mayo RC, Klucznik R, Zhang YJ: Middle cerebral artery aneurysms: a singlecenter series comparing endovascular and surgical treatment. World Neurosurg 81:322-329, 2014

9. Doerfler A, Wanke I, Goericke SL, Wiedemayer H, Engelhorn T, Gizewski ER, et al: Endovascular treatment of middle cerebral artery aneurysms with electrolytically detachable coils. AJNR Am J Neuroradiol 27:513-520, 2006

10. Ewald C, Kühne D, Hassler W: Giant basilar artery aneurysms encorporating the posterior cerebral artery: bypass surgery and coil occlusion-two case reports. Neurol Med Chir (Tokyo) 38 Suppl:83-85, 1998

11. Guglielmi G, Viñuela F, Duckwiler G, Jahan R, Cotroneo E, Gigli R: Endovascular treatment of middle cerebral artery aneurysms. Overall perioperative results. Apropos of 113 cases. Interv Neuroradiol 14:241-245, 2008

12. Güresir E, Schuss P, Berkefeld J, Vatter H, Seifert V: Treatment results for complex middle cerebral artery aneurysms. A prospective single-center series. Acta Neurochir (Wien) 153:1247-1252, 2011

13. Horowitz M, Gupta R, Gologorsky Y, Jovin T, Genevro J, Levy E, et al: Clinical and anatomic outcomes after endovascular coiling of middle cerebral artery aneurysms: report on 30 treated aneurysms and review of the literature. Surg Neurol 66:167-171,2006

14. Iijima A, Piotin M, Mounayer C, Spelle L, Weill A, Moret $\mathrm{J}$ : Endovascular treatment with coils of 149 middle cerebral artery berry aneurysms. Radiology 237:611-619, 2005

15. Kadkhodayan Y, Delgado Almandoz JE, Fease JL, Scholz JM, Blem AM, Tran K, et al: Endovascular treatment of 346 middle cerebral artery aneurysms: results of a 16-year singlecenter experience. Neurosurgery 76:54-61, 2015

16. Kim BM, Kim DI, Park SI, Kim DJ, Suh SH, Won YS: Coil embolization of unruptured middle cerebral artery aneurysms. Neurosurgery 68:346-354, 2011

17. Kim KH, Cha KC, Kim JS, Hong SC: Endovascular coiling of middle cerebral artery aneurysms as an alternative to surgical clipping. J Clin Neurosci 20:520-522, 2013

18. Morgan MK, Mahattanakul W, Davidson A, Reid J: Outcome for middle cerebral artery aneurysm surgery. Neurosurgery 67:755-761, 2010

19. Moroi J, Hadeishi H, Suzuki A, Yasui N: Morbidity and mortality from surgical treatment of unruptured cerebral aneurysms at Research Institute for Brain and Blood VesselsAkita. Neurosurgery 56:224-231, 2005

20. Nanda A, Ambekar S, Sharma M: Surgical management of middle cerebral artery aneurysms. J La State Med Soc 166:160-167, 2014

21. Nussbaum ES, Madison MT, Myers ME, Goddard J: Microsurgical treatment of unruptured intracranial aneurysms. A consecutive surgical experience consisting of 450 aneurysms treated in the endovascular era. Surg Neurol 67:457-466, 2007

22. Ogilvy CS, Crowell RM, Heros RC: Surgical management of middle cerebral artery aneurysms: experience with transsylvian and superior temporal gyrus approaches. Surg Neurol 43:15-24, 1995

23. Quadros RS, Gallas S, Noudel R, Rousseaux P, Pierot L: Endovascular treatment of middle cerebral artery aneurysms as first option: a single center experience of 92 aneurysms. AJNR Am J Neuroradiol 28:1567-1572, 2007

24. Raftopoulos C, Goffette P, Vaz G, Ramzi N, Scholtes JL, Wittebole X, et al: Surgical clipping may lead to better results than coil embolization: results from a series of 101 consecutive unruptured intracranial aneurysms. Neurosurgery 52:1280-1290, 2003

25. Regli L, Uske A, de Tribolet N: Endovascular coil placement compared with surgical clipping for the treatment of unruptured middle cerebral artery aneurysms: a consecutive series. J Neurosurg 90:1025-1030, 1999

26. Rinne J, Hernesniemi J, Niskanen M, Vapalahti M: Analysis of 561 patients with 690 middle cerebral artery aneurysms: anatomic and clinical features as correlated to management outcome. Neurosurgery 38:2-11, 1996

27. Rodríguez-Hernández A, Sughrue ME, Akhavan S, Habdank-Kolaczkowski J, Lawton MT: Current management of middle cerebral artery aneurysms: surgical results with a "clip first" policy. Neurosurgery 72:415-427, 2013

28. Smith TR, Cote DJ, Dasenbrock HH, Hamade YJ, Zammar SG, El Tecle NE, et al: Comparison of the efficacy and safety of endovascular coiling versus microsurgical clipping for unruptured middle cerebral artery aneurysms: a systematic review and meta-analysis. World Neurosurg 84:942-953, 2015

29. Suzuki J, Yoshimoto T, Kayama T: Surgical treatment of middle cerebral artery aneurysms. J Neurosurg 61:17-23, 1984

30. Suzuki S, Tateshima S, Jahan R, Duckwiler GR, Murayama Y, Gonzalez NR, et al: Endovascular treatment of middle cerebral artery aneurysms with detachable coils: angiographic and clinical outcomes in 115 consecutive patients. Neurosurgery 64:876-889, 2009

31. Taha MM, Nakahara I, Higashi T, Iwamuro Y, Iwaasa M, Watanabe Y, et al: Endovascular embolization vs surgical clipping in treatment of cerebral aneurysms: morbidity and mortality with short-term outcome. Surg Neurol 66:277284, 2006

32. van Dijk JMC, Groen RJM, Ter Laan M, Jeltema JR, Mooij JJA, Metzemaekers JDM: Surgical clipping as the preferred treatment for aneurysms of the middle cerebral artery. Acta Neurochir (Wien) 153:2111-2117, 2011

33. Vendrell JF, Menjot N, Costalat V, Hoa D, Moritz J, Brunel $\mathrm{H}$, et al: Endovascular treatment of 174 middle cerebral artery aneurysms: clinical outcome and radiologic results at long-term follow-up. Radiology 253:191-198, 2009

34. Yang W, Huang J: Treatment of middle cerebral artery (MCA) aneurysms: a review of the literature. Chinese Neurosurg J 1:1, 2015

\section{Disclosures}

The authors report no conflict of interest concerning the materials or methods used in this study or the findings specified in this paper.

\section{Author Contributions}

Conception and design: ES Nussbaum, Madison, Goddard, Lassig, LA Nussbaum. Acquisition of data: ES Nussbaum, Madison, Goddard, Lassig, LA Nussbaum. Analysis and interpretation of data: ES Nussbaum, Kallmes, LA Nussbaum. Drafting the article: all authors. Critically revising the article: all authors. Reviewed submitted version of manuscript: all authors. Approved the final version of the manuscript on behalf of all authors: ES Nussbaum. Administrative/technical/material support: Kallmes. Study supervision: ES Nussbaum, Kallmes.

\section{Correspondence}

Eric S. Nussbaum: National Brain Aneurysm Center, Minneapolis, MN.1nussbaum@comcast.net. 